\title{
Simple Use Case Evaluation Method Determining an EHR
}

\section{Integration Platform Design}

\author{
Daniel Krsička ${ }^{1}$, Milan Šárek ${ }^{2}$ \\ ${ }^{1}$ First Faculty of Medicine, Charles University in Prague, Czech Republic \\ ${ }^{2}$ CESNET z.s.p.o., Prague, Czech Republic
}

\begin{abstract}
Integration platform is a basic technical tool for realizing an interoperable Electronic Health Record (EHR). Our goal is to interrelate the knowledge about interoperability, the functions required for an EHR system and the formalized best practises for an integration platform. An evaluation method has been developed, testing dependencies between EHR use cases and logic implemented in the integration platform has been tested on the HL7 EHR System Functional Model.
\end{abstract}

\section{Correspondence to:}

\section{Daniel Krsička}

First Faculty of Medicine, Charles University in Prague

Address: Kateřinská 32, Prague 2, Czech Republic

E-mail: dkrsicka@gmail.com

A dependency has been identified and is discussed in this article.

\section{Keywords}

Interoperability Levels, Electronic Health Record, Healthcare Information System, Integration Platform, Integration Pattern, HL7 EHR System Functional Model

EJBI 2013; 9(3):17-21

recieved: August 15, 2013

accepted: October 28, 2013

published: November 20, 2013

\section{Introduction}

Massive penetration of Healthcare Information Systems (HIS) and eHealth resources in general the significance of Electronic Health Record (EHR) interoperability as an ability of two or more subjects to achieve a common goal or mutually support each other to achieve the individual goals respectively (synergic effect). Theoretical value can be expresses using the Metcalf's Law as the number of all possible connections among subscribers (HIS in our case). It can be asymptotically approximated by the quadratic polynomial of $n 2$. Nevertheless the value of integrated HISs as a whole is not growing quadratic [1]. The HIS integration is not first the establishment of connections between HIS components. It is necessary to pinpoint and follow many protocols enabling an information interchange for particular HIS components and layers. That implies the definition of interoperability level.

Well known authors define several levels of interoperability and its maturity 2, 3. The comparison between levels defined is in Table 1

Our motivation is based on lessons learned about the technological interoperability insufficiency as a means of massive dissemination of interoperable EHR including all needed attributes. This statement is supported by profes- sional publications focusing mainly on EHR system content and semantics. We have published the technological interoperability view inadequacy in [5] and [6]. We have demonstrated that the higher interoperability levels cannot be assured by and based on accepted and broadly used classification into technical layers according to ISO/OSI model in ISO/IEC:7498 [7. The process and partly the semantic interoperability do not have any technical equivalent in ISO/OSI model, so these interoperability levels cannot be procured by technical resources only.

To reach the highest interoperability level is not necessary and should not be an automatic goal for each HIS, because not all the EHR system has to implement all the possible functions.

\subsection{Hypothesis}

Let us suppose that there is a mapping, assigning for each EHR use case an interoperability level required for its realization in a comprehensive EHR system including an integration platform. Evaluating a set of EHR use cases we will get a view on interoperability levels needed and we can use this approach for analysis and design of EHR integration platforms. The benefit is a software analysis simplification and EHR integration platform design op- 
Table 1: Comparison of interoperability levels defined by other authors.

\begin{tabular}{cc}
\hline Levels after Bloebel & Levels after Gibbons \\
\hline Process / Service & Process \\
Semantic & Semantic \\
Syntactic & Technical \\
Structural & Technical \\
Technological & Technical \\
\hline
\end{tabular}

timization. Creating an integration solution among 2 or more EHR systems, the method mentioned below should support analysis and design acceleration, implementation shortening, support of early prototype creation and anticipated decrease of the number of change request, so reducing the total solution costs.

\section{Methods}

We have developed a simple method evaluating every EHR use case in various dimensions. We have evaluated all the use cases for the EHR system defined in HL7 EHR System Functional Model [11. This model serves as a input set of testing data for the method presented here due to we do not have any input data originating from the real EHR integration platform implementation. It is very hard to find some technical solution and gain access to the business analysis outcomes due to almost all the solutions are commercial in the Czech environment.

\subsection{Interoperability Levels and Integration Platform Logical Parts}

We have also defined a mapping between interoperability levels and various integration patterns. Using this mapping we are theoretically able to define which integration patterns (EHR integration platform logical functions) are needed for particular EHR use cases set. The description of individual integration platform layers is out of the scope of this article, so we introduce the list of patterns in each layer only. More information about specific patterns can be found in [8], providing a consistent vocabulary and visual notation to describe large-scale integration solutions across many implementation technologies. Considering the purpose and the added value of each pattern, they can be divided into following groups according to the interoperability level or rather the logical level of the technological solution (integration platform):

- Access Layer Integration Patterns: Channel Adapter, Competing Consumers, Correlation Identifier, Durable Subscriber, Event-driven Consumer, Idempotent Receiver, Message, Message Channel, Message Endpoint, Message Expiration, Messaging Gateway, Polling Consumer, Selective Consumer, Service Activator, Transactional Client,

- Transport Layer Integration Patterns: Channel Purger, Composed Message Channel, Document Message, File Transfer, Guaranteed Delivery, Message Bus, Message Dispatcher, Message Filter, Message Translator, Messaging, Messaging Bridge, Point-to-Point Channel, Publish / Subscribe Channel, Recipient List, Remote Invocation, Request / Reply, Return Address, Shared Database,

- Transformation and Routing Layer Integration Patterns: Aggregator, Content Filter, Dynamic Router, Format Indicator, Message Sequence, Message Router, Resequencer, Splitter

Table 2: EHR use case evaluation criterion: Space, answering questions: "Where the information communication takes place? How distant the points of presence are?"

\begin{tabular}{ll}
\hline Score & Description \\
\hline 0 & Integration in a work team only \\
1 & Integration in one organization, mostly in one location \\
2 & Integration among 2 or more organizations and/or locations \\
\hline
\end{tabular}

Table 3: EHR use case evaluation criterion: Time, answering questions: "When the communication takes place? How fast and often it runs?"

\begin{tabular}{ll}
\hline Score & Description \\
\hline 0 & In real time / mostly "on-line" \\
1 & On daily bases (once or more times a day - hours) \\
2 & One or more times in a month (days / weeks \\
\hline
\end{tabular}


Table 4: EHR use case evaluation criterion: Subject, answering questions: "Who is communicating? What are the subject's skills?"

\begin{tabular}{ll}
\hline Score & Description \\
\hline 0 & Actors with practically the same knowledge and / or education (physicians) \\
1 & Actors with a similar knowledge (physician and nurse) \\
2 & Actors with completely different knowledge (physician and patient) \\
\hline
\end{tabular}

Table 5: EHR use case evaluation criterion: Object, answering questions: "What is communicated? Why runs the communication? For what purpose?"

\begin{tabular}{ll}
\hline Score & Description \\
\hline 0 & Information with common syntax (sharing data) \\
1 & Information with common semantics (sharing information) \\
2 & Information for a deterministic action (sharing knowledge and skills) \\
\hline
\end{tabular}

- Semantic Layer Integration Patterns: Canonical Data, Command Message, Content Enricher, Content-based Router, Claim Check, Datatype Channel, Envelope Wrapper, Event Message, Invalid Message, Messaging Mapper, Normalizer, Test Message

- Business Processes Layer Integration Patterns: Control Bus, Dead Letter Channel, Detour, Message Broker, Message History, Message Store, Pipes and Filters, Process Manager, Routing Slip, Smart Proxy, Scatter / Gather, Wire Tap

\subsection{EHR Use Cases Classification}

The core of presented method is a classification of each EHR use case from 4 different points of view. Each view focuses on different concept. Due to limited space, we cannot describe the method details, including its continual evolution. So we present only an overview. Inspired also by the HL7v3 Reference Information Model [9] and the law of 5W (Who, What, Where, When and Why) [10] we have proposed following classification criterions (see Tables 2, 3, 4, and 5.

Deploying the interoperability levels defined in [2], each EHR use case can get 0 to 8 points in total (4 criterions, 0 - 2 points in each criterion). The most important factor is the sum of score, determining the interoperability level needed for the use case. In case of sum equal 2 we propose a consideration of particular criterion values. If there is leastwise one score of 2 in one criterion, the target level should be syntactic interoperability. A score evaluation overview is in Table 6.

\subsection{Experiments - Model EHR Use Cases and Interoperability}

We have applied the aforementioned method on 64 functions required in HL7 EHR-S Functional Model [11. For definition of use cases, we have assumed that these functions have to be realized in a common hospital. This way we can anticipate actors and other factor needed for the instantiation of a function (use case definition). An example of the use cases evaluation is in Table 7

\section{Results}

Aggregating all of the 64 experiments we can summarize that the interoperability level needed for implementing all the functions in the HL7 EHR System Functional Model [11. It means that we have used each function from this model, transform it into the EHR Information System Use Case and have applied the method presented here. This application results into a cumulative sets of values indicating the most intensive interoperability level needed to solve in the integration platform solution design. Results in graphics can be in Figure 1, the vertical axis represents the number of incidences, the horizontal axis depicts the score attained during the method application for each Use Case.

Table 6: Total score determining the target level of interoperability.

\begin{tabular}{cc}
\hline Score attained (sum) & Target interoperability level \\
\hline $0-2$ & Technical / Structural \\
$2-3$ & Syntactic \\
$4-5$ & Semantic \\
$6-8$ & Process / Service \\
\hline
\end{tabular}


Table 7: A sample of evaluation of use cases derived from HL7 EHR-S Functional Model, section Direct Care (DC).

\begin{tabular}{lccccc}
\hline Function ID used & Space & Time & Subject & Object & Sum \\
\hline DC.1.1.1 & 1 & 0 & 2 & 0 & 3 \\
DC.1.1.2 & 1 & 1 & 2 & 1 & 5 \\
DC.1.1.3.1 & 2 & 1 & 1 & 1 & 5 \\
DC.1.1.3.2 & 2 & 1 & 2 & 0 & 5 \\
DC.1.1.3.3 & 2 & 2 & 2 & 0 & 6 \\
\hline
\end{tabular}

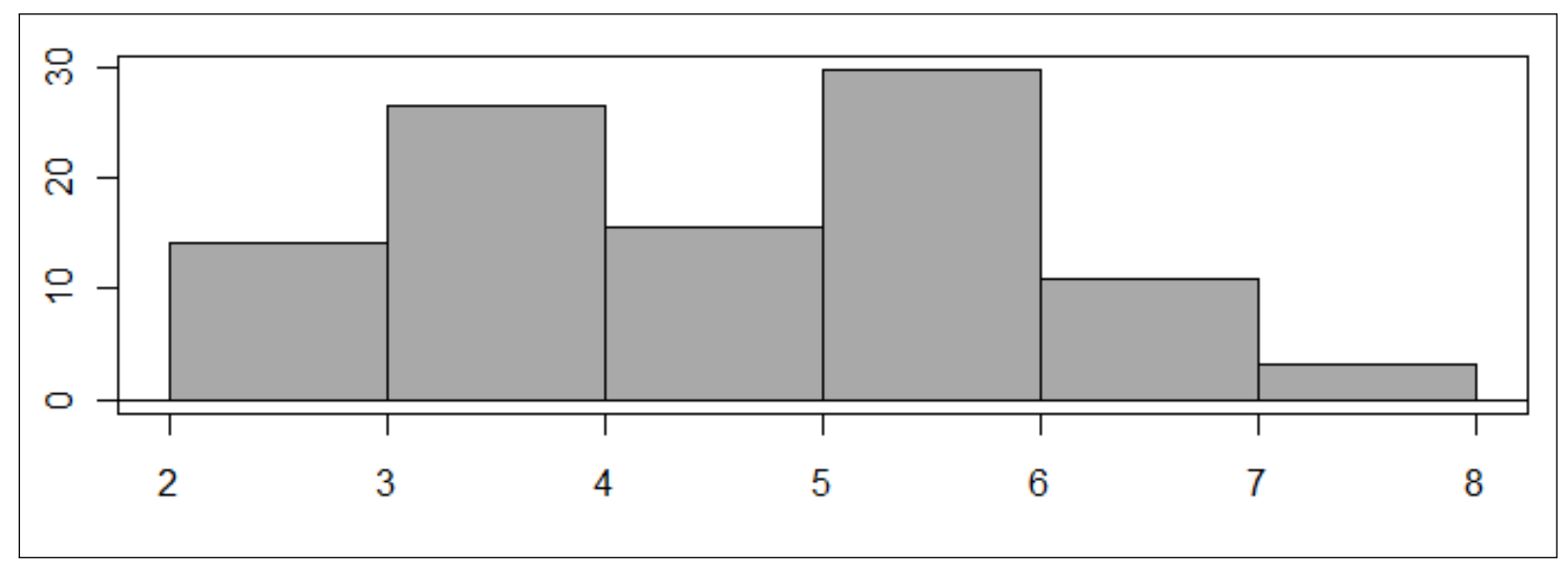

Figure 1: Histogram with results of HL7 EHR-S Functional Model use cases.

It is clear that the majority of use cases evaluation scores does not exceed the value of 5 . It means that the target interoperability for all the use cases derived from the HL7 EHR System Functional Model is at most the semantic interoperability. Of course the condition of semantic interoperability is the usage and implementation of all the lower interoperability levels. The integration patterns mentioned in 2.1 corresponding to these levels should be used.

On the other hands any investment into technologies and platforms supporting integration patterns related to the process interoperability level should be considered in detail.

\section{Discussion}

The presented method has been applied to 64 HL7 EHR-S Functional Model uses cases derived from [11. The understanding of these rules is quite simple, so the use cases can be evaluated also by a person without a specialized training in computer science and software engineering (physician, manager ...). It offers the possibility to bridge the interdisciplinary gap among different actors. This way, a mapping between different Generic Component Model domains [12] is enabled in the integration platform development process.

The method implies the possibility of a structured view to the often heterogeneous set of (business) requirements. It has to be tested, whether the method can really simplify the analysis project phase and enable the development of an early integration platform prototype. The benefit of early prototyping is the possibility to test soon after the requirement specification, to decrease the number of change requests, to speed up the project, and to lower the total costs.

\section{Conclusion}

It seems that the method presented here should accelerate an EHR integration platform analysis and design and save time and costs in this way. The second, but not least, benefit lies in the possibility of gap bridging between various roles interested in above mentioned EHR software analysis and design. But there is one very important condition. The method must be tested on the real EHR integration use cases and only then we can compare the existing EHR integration designed in the traditional way with the design emerging from our method, assess its reliability and continually work on its optimization.

\section{Acknowledgements}

The paper has been supported by the SVV-2013-266 517 project of Charles University in Prague.

\section{References}

[1] Benson, T. Principles of health interoperability HL7 and SNOMED. New York: Springer, 2012, ISBN 978-144-7128-007.

[2] Bloebel, B. Architectural Approach to eHealth for Enabling 
Paradigm Changes in Health. Methods of Information in Medicine. 2010, 49, s.123-134.

[3] Gibbons, P. Coming to Terms: White Paper on Interoperability. In: HL7 [online]. 2007 [cit. 2012-0814]. Via: http://www.hl7.org/documentcenter/public/wg/ehr /ComingtoTerms2007-03-22.zip

[4] ISO/EN 13606 Health informatics - Electronic health record communication. Geneva, Switzerland: International Organization for Standardization, 2008-2010.

[5] Krsicka, D. a M. Sarek. Automatizace vyuziti blokovych reseni pro vyvoj architektur IS. In: MEDSOFT 2012. Praha: Dum techniky CSVTS, 2012, s. 168-179. ISSN 1803-8115.

[6] Krsicka, D. a M. Sarek. Integracni vzory a jejich automaticke vyhodnocovani. In: Medsoft 2011. Praha: Creative Connections, 2011, s. 146-149. ISSN 1803-8115.

[7] ISO/IEC 7498-1:1994. Information technology - Open Systems Interconnection: Basic Reference Model: The Basic Model.
Geneva, Switzerland: International Organization for Standardization, 1997.

[8] Hohpe, G. Enterprise integration patterns: designing, building, and deploying messaging solutions. Boston: AddisonWesley, 2004, 683 s. ISBN 03-212-0068-3.

[9] Health Level Seven International:HL7 [online]. 2012 [cit. 201206-19]. Via: http://www.hl7.org

[10] Five Ws. In: Wikipedia: the free encyclopedia [online]. San Francisco (CA): Wikimedia Foundation, 2001- [cit. 2012-0815]. Via: http://en.wikipedia.org/wiki/Five_Ws

[11] HL7 EHR System Functional Model R1.1. HL7. HL7 [online]. 2012 [cit. 2012-10-29]. From http://www.hl7.org/implement/standards/product brief.cfm?product id $=269$

[12] Bloebel, B., Oemig, F. What Is Needed to Finally Achieve Semantic Interoperability? In: Doessel, O., Schlegel, W. C. (Edrs.) IFMBE Proceedings 25/XII. 2012, p. 411-415 